\title{
Preconditioning Iterative MLFMA Solutions of Integral Equations
}

\author{
Levent Gürel ${ }^{\# 1}$, Tahir Malas ${ }^{\# 2}$, and Özgür Ergül ${ }^{* 3}$ \\ \# Department of Electrical and Electronics Engineering \\ Computational Electromagnetics Research Center (BiLCEM) \\ Bilkent University, TR-06800, Ankara, Turkey \\ * Department of Mathematics and Statistics \\ University of Strathclyde, G11XH, Glasgow, UK \\ ${ }^{1}$ Igurel@bilkent.edu.tr, ${ }^{2}$ tmalas@ee.bilkent.edu.tr, 3ozgur.ergul@strath.ac.uk
}

\begin{abstract}
The multilevel fast multipole algorithm (MLFMA) is a powerful method that enables iterative solutions of electromagnetics problems with low complexity. Iterative solvers, however, are not robust for three-dimensional complex reallife problems unless suitable preconditioners are used. In this paper, we present our efforts to devise effective preconditioners for MLFMA solutions of difficult electromagnetics problems involving both conductors and dielectrics.
\end{abstract}

\section{INTRODUCTION}

Sequential and parallel solutions of integral equations with Krylov subspace iterative solvers and the multilevel fast multipole algorithm (MLFMA) have been very useful for largescale computational electromagnetics (CEM) problems [1] [4]. Iterative solvers, however, usually need preconditioners for the solution of large and difficult problems.

In this paper, we demonstrate our efforts to devise effective preconditioners, both sequential and parallel ones. We consider perfect-electric-conductor (PEC) and dielectric problems. Note that we assume uniform meshes and the high-frequency case. For the low-frequency case, which also allows non-uniformity, Calderón preconditioners can be used [5]-[7].

\section{Conductor Problems}

Geometries involving open surfaces are formulated with the electric-field integral equation (EFIE). It is formed by a physical boundary condition, which states that total tangential electric field vanishes on a conducting surface. With this condition, EFIE can be expressed as

$$
\hat{\boldsymbol{t}} \cdot \int_{S^{\prime}} \mathrm{d} \mathbf{r}^{\prime} \overline{\boldsymbol{G}}\left(\mathbf{r}, \mathbf{r}^{\prime}\right) \cdot \boldsymbol{J}\left(\mathbf{r}^{\prime}\right)=\frac{i}{k \eta} \hat{\boldsymbol{t}} \cdot \boldsymbol{E}^{i n c}(\mathbf{r}),
$$

where $\boldsymbol{E}^{i n c}$ represents the incident electric field, $S^{\prime}$ is the surface of the object, $\hat{t}$ is any tangential unit vector on $S^{\prime}$, $\boldsymbol{J}\left(\mathbf{r}^{\prime}\right)$ is the unknown induced current residing on the surface, $\bar{G}\left(\mathbf{r}, \mathbf{r}^{\prime}\right)$ is the dyadic Green's function.

Similarly, the boundary condition for the tangential magnetic field on a conducting surface is used to derive the magnetic-field integral equation (MFIE) as

$$
\boldsymbol{J}(\mathbf{r})-\hat{\boldsymbol{n}} \times \int_{S^{\prime}} \mathrm{d} \mathbf{r}^{\prime} \boldsymbol{J}\left(\mathbf{r}^{\prime}\right) \times \nabla^{\prime} g\left(\mathbf{r}, \mathbf{r}^{\prime}\right)=\hat{\boldsymbol{n}} \times \boldsymbol{H}^{i n c}(\mathbf{r}),
$$

where $\hat{\boldsymbol{n}}$ is any unit normal on $S^{\prime}$ and $\boldsymbol{H}^{i n c}(\mathbf{r})$ is the incident magnetic field. We note that MFIE is valid only for closedsurface problems.

Combining EFIE and MFIE, we obtain the combined-field integral equation (CFIE), i.e.,

$$
\mathrm{CFIE}=\alpha \mathrm{EFIE}+(1-\alpha) \mathrm{MFIE},
$$

where we choose $\alpha$ in the $0.2-0.3$ range.

Upon the discretization of (1), (2), or (3) by the method of moments, we end up with a dense linear system. The surface of the objects are in general meshed with 1/10th of the wavelength for accuracy. Hence, for high frequencies where the scatterer or the radiator sizes become large in terms of the wavelength, the system matrix becomes also large.

When iterative methods are used to solve such systems, they can at best provide $\mathcal{O}\left(N^{2}\right)$ complexity. This is prohibitive for large problems. Hence, the solutions of such problems is viable only with fast methods such as MLFMA, which drops the complexity of the dense matrix-vector multiplication to $\mathcal{O}(N \log N)$.

MLFMA is proposed as a multilevel extension of the singlelevel fast multipole method. In order to perform interactions between the basis and testing functions in a group-by-group manner, the whole geometry is placed into a cube and it is recursively divided into smaller ones until the smallest cubes contain only a few basis functions. During the partitioning, if any of the cubes become empty, recursion stops there. MLFMA replaces element-to-element interactions with cluster-to-cluster interactions in a multilevel scheme. This computational scheme relies on the factorization of the Green's function, which is valid only for basis and testing functions that are far from each other. In the lowest level, interactions between the near-field clusters are computed directly and stored in the sparse matrix $\bar{Z}^{\mathrm{NF}}$. Interactions among the farfield clusters are computed approximately but with controllable error. For this purpose, the radiated fields of each cluster are aggregated at the centers of the clusters. Then, for each pair of far-field clusters whose parents are near to each other, cluster-to-cluster interaction is computed via a translation. Finally, after the translations, the matrix-vector multiplication is completed by disaggregating the incoming fields to the 
centers of the testing clusters and onto the testing functions. We refer the readers to [4] for the details of the parallelization of MLFMA.

\section{NEAR-FIELD PRECONDITIONERS}

It is customary to construct preconditioners from the readily available sparse matrix $\bar{Z}^{\mathrm{NF}}$, assuming that it is a good approximation to $\overline{\boldsymbol{A}}$. We group these preconditioners as the block-diagonal preconditioner, incomplete factorization methods, sparse approximate inverses (SAIs), and the iterative nearfield scheme.

\section{A. The Block-Diagonal Preconditioner}

This is the most widely used preconditioner for CFIE in CEM community. The block-diagonal preconditioner is usually constructed from the self-interactions of the lowest-level clusters. Even though it has low setup time, for complex and closed targets, stronger preconditioners has a good potential to improve the convergence rate [8]. For EFIE systems, this preconditioner deteriorates the convergence rate compared to the no-preconditioning case, hence it should not be used.

\section{B. Incomplete LU Preconditioners}

Incomplete factorization methods are the most popular and widely used preconditioners in scientific computing. They are based on eliminating some of the entries of the lower and upper matrices in an LU factorization [9]. In MLFMA context, after decomposing the near-field matrix in the form of $\overline{\boldsymbol{A}}^{\mathrm{NF}} \approx$ $\overline{\boldsymbol{L}} \cdot \overline{\boldsymbol{U}}$, preconditioning operation is performed in each step by solving $\overline{\boldsymbol{L}} \cdot \overline{\boldsymbol{U}} \cdot \boldsymbol{v}=\boldsymbol{w}$, where $\overline{\boldsymbol{L}}$ and $\overline{\boldsymbol{U}}$ are the incomplete factors.

The most widely used ILU-type preconditioner is the nofill ILU, or ILU(0). It is obtained by retaining the nonzero values of $\overline{\boldsymbol{L}}$ and $\overline{\boldsymbol{U}}$ only at the nonzero positions of $\overline{\boldsymbol{Z}}^{\mathrm{NF}}$. For well-conditioned systems that are not far from being diagonally dominant, this simple idea works well. Moreover, ILU(0) has a very low setup time compared to other ILU-type preconditioners.

For more difficult problems, the ILUT preconditioner is known to yield more accurate factorizations compared to ILU(0) with the same amount of fill-in [9]. ILUT uses two parameters: a threshold $\tau$ and the maximum number of nonzero elements per row $p$. During the factorization, matrix elements that are smaller than $\tau$ times 2-norm of the current row are dropped. Then, of all the remaining entries, no more than the $p$ largest ones are kept. In MLFMA context, we set the threshold $\tau$ to a low value such as $10^{-4}$ and choose $p$ so that the preconditioner uses the same amount of memory as the near-field matrix.

However, ILUT can still fail due to the stability problems. A measure for the stability can be achieved by using the condition estimate of the incomplete factors, which is called condest. This metric can be found by

$$
\left\|(\overline{\boldsymbol{L}} \cdot \overline{\boldsymbol{U}})^{-1} \cdot \boldsymbol{e}\right\|_{\infty}
$$

where $e$ is the vector of ones. If the condest value is very high, we can deduce that there is an instability issue and try pivoting to remedy the situation. The resulting preconditioner is called ILUTP.

In our previous work [8], we showed that for CFIE, ILU(0) provides a cheap but very close approximation to the near-field matrix; hence it reduces the iteration counts and solution times substantially compared to the block-diagonal preconditioner. For ill-conditioned EFIE matrices, however, we showed the need to use a more robust ILUT and apply pivoting whenever required.

\section{Sparse Approximate Inverses}

Contrary to ILU preconditioners, a SAI $\bar{M}$ directly approximates the inverse of the matrix. Then, application of the preconditioner is performed simply with the sparse-matrix vector multiplication $\boldsymbol{v}=\overline{\boldsymbol{M}} \cdot \overline{\boldsymbol{U}} \cdot \boldsymbol{w}$. The backward and forward substitutions required in the incomplete factorization methods are inherently sequential; hence for parallel applications approximate inverse type preconditioners are preferred.

There are various types of SAI preconditioners. Among them, the one that is based on Frobenius norm minimization is successfully used in CEM problems for EFIE [10]. We note that, SAI has a good potential to be helpful for real-life problems formulated by CFIE [11].

For the SAI preconditioner that depends on Frobenius norm minimization, the sparsity pattern of the approximate inverse should be prescribed. When, the same pattern of $\bar{Z}^{\mathrm{NF}}$ is used for the approximate inverse, significant reduction can be achieved in setup time, because of the block-structure of the near-field matrix [10]. However, filtering may be adequate to gain from memory sometimes.

After determining the sparsity pattern of the preconditioner, the approximate inverse of the near-field matrix is performed by minimizing

$$
\left\|\overline{\boldsymbol{I}}-\overline{\boldsymbol{M}} \cdot \overline{\boldsymbol{Z}}^{\mathrm{NF}}\right\|_{F}
$$

For a row-wise parallel decomposition scheme, minimization can be performed independently for each row by using the identity

$$
\left\|\overline{\boldsymbol{I}}-\overline{\boldsymbol{M}} \cdot \overline{\boldsymbol{Z}}^{\mathrm{NF}}\right\|_{F}^{2}=\sum_{i=1}^{N}\left\|\boldsymbol{e}_{\boldsymbol{i}}-\boldsymbol{m}_{\boldsymbol{i}} \cdot \overline{\boldsymbol{Z}}^{\mathrm{NF}}\right\|_{2}^{2},
$$

where $\boldsymbol{e}_{\boldsymbol{i}}$ is the $i$ th unit row vector and $\boldsymbol{m}_{\boldsymbol{i}}$ is the $i$ th row of the preconditioner.

\section{Iterative Near-Field Preconditioner}

For ill-conditioned problems such as those produced by EFIE, it is known that SAI is not as successful as ILU when we use the same amount of memory [12]. On the other hand, since SAI is a good approximation to the inverse of the nearfield matrix, a fast iterative solution of the system involving near-field matrix can be obtained and used as a preconditioner. This approach produces a nested implementation of iterative solvers. In the outer solver that solves the original system, we use FGMRES, a flexible version of GMRES, which allows 
the preconditioner to change from iteration to iteration. Then, the preconditioner of this solver can be another preconditioned Krylov subspace solver which is called the inner solver. We solve the near-field system in the inner solver, using SAI as the fixed preconditioner. We illustrate this preconditioning scheme in Figure 1.

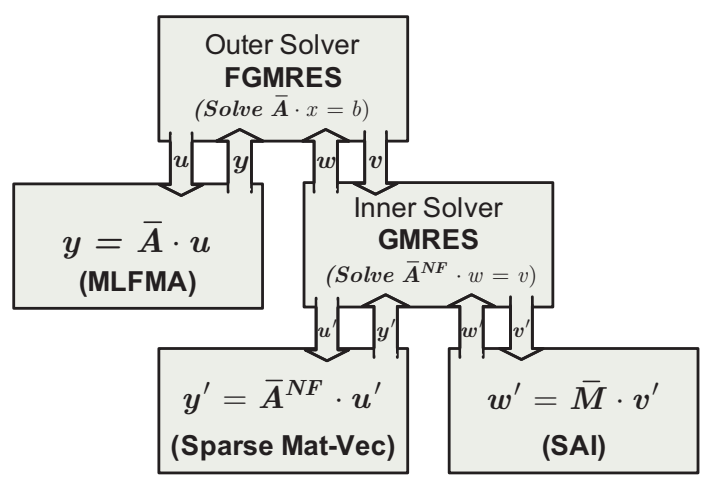

Fig. 1. Graphical representation of the INF preconditioner.

Since the inner solver is used for preconditioning purposes, a rough solution can be adequate. Hence, GMRES is a suitable choice for the inner solver since it provides a fast drop of the residual norm in the early iterations.

\section{Approximate Full-Matrix Preconditioners}

The usual practice in MLFMA is to keep the size of the lowest-level clusters fixed and to partition the target in a bottom-up fashion. Hence, as the problem size and the number of MLFMA levels increase, the near-field matrix becomes sparser. Therefore, for large-scale problems, we may need more than what is provided by the near-field matrix.

When we have the opportunity to use an iterative procedure for preconditioning, as in the iterative near-field scheme, we can make use of MLFMA to have stronger preconditioners than those obtained from the near-field matrix. In order to reduce the solution time, inexpensive versions of MLFMA can be introduced and used for the inner solver.

In MLFMA, the maximum error is controlled by the truncation number

$$
L \approx 1.73 k a+2.16\left(d_{0}\right)^{2 / 3}(k a)^{1 / 3}
$$

of the translation function, where $a$ is the cluster size of the level and $d_{0}$ is the number of accurate digits [13]. In order to balance the accuracy and efficiency in a flexible way, we redefine the truncation number for level $l$ as

$$
L_{l}^{\prime}=L_{1}+a_{f}\left(L_{l}-L_{1}\right),
$$

where $L_{1}$ is the truncation number defined for the first level, $L_{l}$ is the original truncation number for the level $l$ calculated by using (7). The approximation factor $a_{f}$ is defined in the range from 0.0 to 1.0. As $a_{f}$ increases from 0.0 to 1.0 , the AMLFMA becomes more accurate but less efficient, while it corresponds to the full MLFMA when $a_{f}=1$. Hence, this parameter provides us important flexibility in designing the preconditioner. Moreover, the truncation number of the lowest level is not modified, hence AMLFMA does not require extra computation load for the radiation and receiving patterns of the basis and testing functions when it is used in conjunction with MLFMA in a nested manner.

\section{Dielectric Problems}

Recently developed surface formulations of dielectric problems increase the stability of the resulting matrix equations, hence they are more suitable for iterative solutions employing MLFMA [14]. Among those formulations, we consider the combined tangential formulation $(\mathrm{CTF})$, which produces more accurate results, and the electric and magnetic current combined-field integral equation (JMCFIE), which produces better-conditioned matrix systems than other formulations.

CTF and JMCFIE can be regarded as the counterparts of EFIE and CFIE, which are commonly used in PEC problems. For real-life problems with high dielectric constants, however, matrix systems resulting from both CTF and JMCFIE represent a significant challenge in terms of convergence because of indefiniteness and poor spectral properties. To overcome this problem, we propose two variants of Schur complement preconditioners that are improved versions of those introduced in [15].

\section{Schur Complement PRECONDitioning}

In order to devise an effective preconditioner for dielectric problems, the $2 \times 2$ partitioned near-field matrix system

$$
\left[\begin{array}{ll}
\overline{\boldsymbol{A}}_{11}^{N F} & \overline{\boldsymbol{A}}_{12}^{N F} \\
\overline{\boldsymbol{A}}_{21}^{N F} & \overline{\boldsymbol{A}}_{22}^{N F}
\end{array}\right] \cdot\left[\begin{array}{l}
\boldsymbol{v}_{1} \\
\boldsymbol{v}_{2}
\end{array}\right]=\left[\begin{array}{l}
\boldsymbol{w}_{1} \\
\boldsymbol{w}_{2}
\end{array}\right]
$$

should be efficiently solved with minimum computational requirements. It is possible to provide fast and yet successful approximations to solutions of such partitioned matrix systems using Schur complement reduction. With this method, the solution of the $2 N \times 2 N$ near-field system in (9) can be reduced into the solutions of $N \times N$ reduced systems

$$
\overline{\boldsymbol{S}} \cdot \boldsymbol{v}_{2}=\boldsymbol{w}_{2}-\overline{\boldsymbol{A}}_{21}^{N F} \cdot\left(\overline{\boldsymbol{A}}_{11}^{N F}\right)^{-1} \cdot \boldsymbol{w}_{\mathbf{1}}
$$

and

$$
\overline{\boldsymbol{A}}_{11}^{N F} \cdot \boldsymbol{v}_{1}=\boldsymbol{w}_{\mathbf{1}}-\overline{\boldsymbol{A}}_{12}^{N F} \cdot \boldsymbol{v}_{2}
$$

where

$$
\overline{\boldsymbol{S}}=\overline{\boldsymbol{A}}_{22}^{N F}-\overline{\boldsymbol{A}}_{21}^{N F} \cdot\left(\overline{\boldsymbol{A}}_{11}^{N F}\right)^{-1} \cdot \overline{\boldsymbol{A}}_{12}^{N F}
$$

is the Schur complement. Approximate solutions to (10) and (11), which can be obtained either directly or iteratively, induce effective preconditioners. The preconditioner that uses the direct approach is called "the approximate Schur preconditioner (ASP)" and the one that uses the iterative approach "the iterative Schur preconditioner (ISP)." When the number of inner iterations for the reduced systems are not fixed, the outer iterative solver employed for ISP should be chosen as a flexible Krylov solver [16].

Both ASP and ISP require approximate inverses of $\overline{\boldsymbol{A}}_{11}^{N F}$ and $\overline{\boldsymbol{S}}$. For ISP, approximate inverses should be used as "inner" 
preconditioners to accelerate iterative solutions of the reduced systems (10) and (11). For $\overline{\boldsymbol{A}}_{11}^{N F}$, we use a sparse approximate inverse (SAI) based on Frobenius-norm minimization, which has proven to yield a successful approach in PEC problems formulated with EFIE or CFIE [11]. This SAI, which we denote by $\overline{\boldsymbol{M}}_{11}$, uses the sparsity pattern of $\overline{\boldsymbol{A}}_{11}^{N F}$. Hence, it costs only one-fourth of the memory consumed by the nearfield matrix. $\overline{\boldsymbol{M}}_{11}$ is also used to approximate the inverse of $\overline{\boldsymbol{A}}_{11}^{N F}$ in the right-hand side of (10) and in matrix-vector multiplications (MVMs) of $\overline{\boldsymbol{S}}$ for ISP.

In the literature, sparse approximations to both $\bar{S}$ itself and the inverse of $\overline{\boldsymbol{S}}$ have been developed when the $(1,1)$ partition is zero or it has a small size [16], which are not the cases for CTF or JMCFIE. As proposed in [15], we can approximate the inverse of $\bar{S}$ by the SAI of $\overline{\boldsymbol{A}}_{11}^{N F}$, which is equal to $\bar{M}_{11}$ due to identical diagonal partitions of CTF or JMCFIE matrices. However, this choice fails to provide a successful approximate inverse for a large dielectric constant, since the near-field matrix loses its diagonal dominance [14]. To include the second term of $\bar{S}$, only diagonal blocks of the partitions can be taken into account, as in [17]. However, this approach also fails in CTF and for large dielectric constants in JMCFIE.

In this paper, we propose a more effective approach. To retain the near-field matrix information beyond the diagonal partitions and the information in the second term of $\overline{\boldsymbol{S}}$, we first compute a sparse approximation to $\bar{S}$ in the form of

$$
\widetilde{\bar{S}}=\overline{\boldsymbol{A}}_{22}^{N F}-\overline{\boldsymbol{A}}_{21}^{N F} \odot \overline{\boldsymbol{M}}_{11} \odot \overline{\boldsymbol{A}}_{12},
$$

where $\odot$ denotes an incomplete matrix-matrix multiplication obtained by retaining the near-field sparsity pattern. Then, the inverse of $\overline{\boldsymbol{S}}$ is approximated by

$$
\overline{\boldsymbol{M}}_{S} \approx \widetilde{\bar{S}}^{-1} \approx \overline{\boldsymbol{S}}^{-1}
$$

where $\overline{\boldsymbol{M}}_{S}$ denotes a SAI of $\widetilde{\overline{\boldsymbol{S}}}$. If the entries of the near-field partitions are stored row-wise, the incomplete matrix-matrix multiplications in (13) can be performed in $\mathcal{O}(N)$ time using the $i k j$ loop order of the matrix-matrix multiplication. The proposed SAI for $\bar{S}$ generates a successful approximation of the inverse of $\bar{S}$ that can be used as a direct solver or as a strong preconditioner.

\section{REFERENCES}

[1] J. Fostier and F. Olyslager, "An Asynchronous Parallel MLFMA for Scattering at Multiple Dielectric Objects," IEEE Trans. Antennas Propagat., vol. 56, pp. 2346-2355, 2008.

[2] J. Fostier and F. Olyslager, "Provably scalable parallel multilevel fast multipole algorithm," Electron. Lett., vol. 44, no. 19, pp. 1111-1113, 2008.

[3] J. Fostier and F. Olyslager, "Full-wave electromagnetic scattering at extremely large 2-D objects," Electron. Lett., vol. 45, no. 5, 2009.

[4] Ö. Ergül and L. Gürel, "Efficient parallelization of the multilevel fast multipole algorithm for the solution of large-scale scattering problems," IEEE Trans. Antennas Propagat., vol. 56, no. 8, pp. 23352345, 2008.

[5] F. P. Andriulli, K. Cools, H. Bag̃cı, F. Olyslager, A. Buffa, S. Christiansen, and E. Michielssen, "A multiplicative Calderón preconditioner for the electric field integral equation," IEEE Trans. Antennas Propagat., vol. 56, no. 8, pp. 2398-2412, 2008.

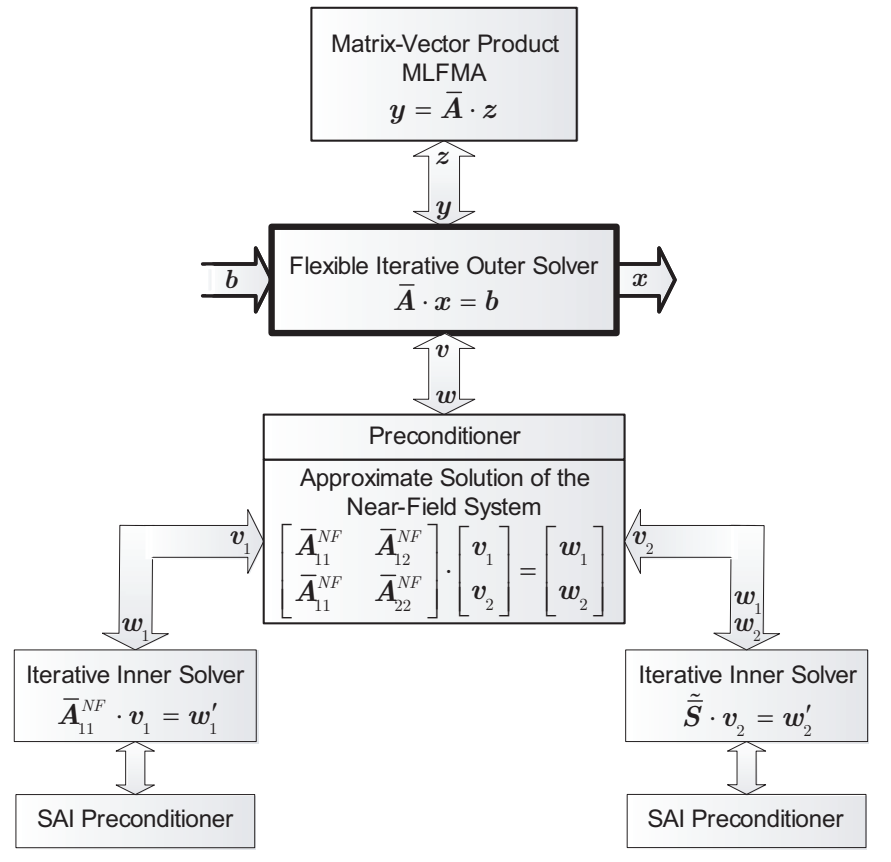

Fig. 2. Illustration of the solution of dielectric problems using MLFMA and iterative Schur complement preconditioners. The matrix $\widetilde{\bar{S}}$ is an approximation to the Schur complement, and $\boldsymbol{w}_{1}^{\prime}$ and $\boldsymbol{w}_{2}^{\prime}$ take different forms depending on the type of the preconditioner.

[6] H. Bag̃ c1, F. P. Andriulli, K. Cools, F. Olyslager, and E. Michielssen, "A Calderón multiplicative preconditioner for the combined field integral equation," IEEE Trans. Antennas Propagat., vol. 57, no. 10, pp. 3387-3392, Oct. 2009.

[7] J. Peeters, K. Cools, I. Bogaert, F. Olyslager, and D. De Zutter, "Embedding Calderón multiplicative preconditioners in multilevel fast multipole algorithms," IEEE Trans. Antennas Propagat., accepted, Jan. 2010.

[8] T. Malas and L. Gürel, "Incomplete LU preconditioning with the multilevel fast multipole algorithm for electromagnetic scattering," SIAM J. Sci. Comput., vol. 29, no. 4, pp. 1476-1494, 2007.

[9] Y. Saad, Iterative Methods for Sparse Linear Systems, 2nd ed. Philadelphia, PA, USA: SIAM, 2003.

[10] B. Carpentieri, I. S. Duff, L. Giraud, and G. Sylvand, "Combining fast multipole techniques and an approximate inverse preconditioner for large electromagnetism calculations," SIAM J. Sci. Comput., vol. 27, no. 3, pp. 774-792, 2005.

[11] T. Malas and L. Gürel, "Accelerating the multilevel fast multipole algorithm with the sparse-approximate-inverse (SAI) preconditioning," SIAM J. Sci. Comput., vol. 31, no. 3, pp. 1968-1984, 2009.

[12] M. Benzi and M. Tuma, "A comparative study of sparse approximate inverse preconditioners," Appl. Numer. Math., vol. 30, no. 2-3, pp. 305-340, 1999.

[13] M. L. Hastriter, S. Ohnuki, and W. C. Chew, "Error control of the translation operator in 3D MLFMA," Microwave Opt. Technol. Lett., vol. 37, no. 3, pp. 184-188, 2003.

[14] P. Ylä-Oijala, M. Taskinen, and S. Järvenpää, "Analysis of surface integral equations in electromagnetic scattering and radiation problems," Eng. Anal. Boundary Elem., vol. 32, no. 3, pp. 196-209, 2008.

[15] T. Malas and L. Gürel, "An effective preconditioner based on the Schur complement reduction for integral-equation formulations of dielectric problems," in IEEE International Symposium on Antennas and Propagation, Charleston, South Carolina, USA, June 2009.

[16] M. Benzi, G. H. Golub, and J. Liesen, "Numerical solution of saddle point problems," Acta Numer., vol. 14, pp. 1-137, 2005.

[17] Ö. Ergül and L. Gürel, "Comparison of integral-equation formulations for the fast and accurate solution of scattering problems involving dielectric objects with multilevel fast multipole algorithm," IEEE Trans. Antennas Propagat., vol. 57, no. 1, pp. 176-187, Jan. 2009. 